\title{
DYSLIPIDAEMIA
}

\section{Promising results for TA-8995 in TULIP}

Many patients with dyslipidaemia remain at high cardiovascular risk despite using statins and other lipid-lowering therapies. Several new LDL-cholesterol-lowering strategies are, therefore, being investigated, including the inhibition of cholesterol ester transfer protein (CETP). Testing of this strategy has provided mixed results to date; however, two CETP inhibitorsanacetrapib and evacetrapib-have been found to exert favourable effects on blood lipids and are currently being tested in large clinical outcome trials. The findings of the phase II TULIP trial of another CETP inhibitor, TA-8995, have now been published in The Lancet. The investigators believe that "the translation of the antiatherogenic potential of TA-8995 ... into a reduction of future cardiovascular events warrants formal testing in a cardiovascular outcome trial".

In the phase II TULIP trial, 364 patients with mild dyslipidaemia were randomly assigned to receive no study drug; $1 \mathrm{mg}$, $2.5 \mathrm{mg}, 5 \mathrm{mg}$, or $10 \mathrm{mg}$ TA-8995 daily, but no statin; $10 \mathrm{mg}$ TA-8995 plus a statin
(20 $\mathrm{mg}$ atorvastatin or $10 \mathrm{mg}$ rosuvastatin) daily; or a statin $(20 \mathrm{mg}$ atorvastatin or $10 \mathrm{mg}$ rosuvastatin) daily, but no CETP inhibitor. Patients who did not receive TA-8995 and/or a statin received matching placebos to maintain masking.

At 12 weeks, LDL-cholesterol levels were significantly reduced and HDL-cholesterol levels were significantly increased with all doses of TA-8995. Patients who received a statin plus the CETP inhibitor had lower LDL-cholesterol levels and higher HDL-cholesterol levels than those who received a statin only. Levels of other blood lipids were also improved with the CETP inhibitor, which was well tolerated.

Bryony M. Mearns

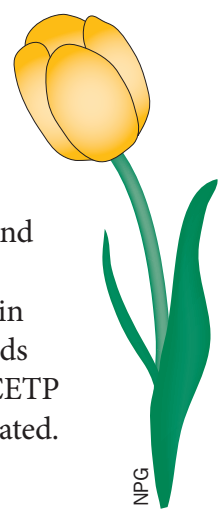

Original article Hovingh, G. K. et al. Cholesterol ester transfer protein inhibition by TA-8995 in patients with mild dyslipidaemia (TULIP): a randomised, double-blind, placebo-controlled phase 2 trial. Lancet doi:10.1016/ S0140-6736(15)60158-1 